Methods

- Steering group identified members confident in videoconferencing, producing supporting guidance for members.

- Discussion topics identified through feedback.

- Staff/volunteer speakers approached by steering group member; subsequent briefing discussion and format explanation.

- Brief online survey emailed to participants.

Results 36 members of staff responded to the survey: 55\% clinical, 45\% non-clinical staff. What participants like about Schwartz:

- Sense of community/connection.

- Non-judgmental space.

- Understanding others' roles.

- Aiding own reflection.

- Learning from others' views.

19 answered 'what could be done differently?' Ten people (from the total of 19) responded to say that they didn't think anything could be done differently. Themes identified were:

- Varying date/time to accommodate different working patterns.

- Taking advance questions.

- Return to physical meetings for easier interaction, especially before session.

Some commented how well an online platform worked, making it accessible to participants offsite. Ideas for future topics: understanding roles; COVID-19's impact; coping with stress.

Conclusion Online SCR transition was largely positively received. Overarching aim to allow people space to reflect, discuss challenging clinical scenarios and receive support. Despite anxiety about ability to connect emotionally remotely, the feedback showed sense of community and non-judgemental space for reflection was not compromised in a potentially isolating time. We aim to alternate face-to-face and remote SCR to improve access for staff/volunteers.

\section{P-194 CLINICAL SUPERVISION IN THE HOSPICE INPATIENT UNIT}

Jamie Yeomans, Sarah Popplestone-Helm. St Richard's Hospice, Worcester, UK

\subsection{6/spcare-2021-Hospice.210}

Background Clinical supervision has been defined as 'an exchange between practicing professionals to enable the development of professional skills' (Faugier \& Butterworth, 1994): Conversely, Hyrkas et al suggested that although clinical supervision is encouraged widely in nursing literature, studies carried out (to the date of their published work) had failed to evidence how supervision benefits clinical practice (Hyrkas, Koivula, Paunomon, 1999). More recent research, however, suggests that clinical supervision supports practice and helps healthcare practitioners maintain and improve standards by reflecting and identifying workable strategies for future work (Sullivan \& Garland, 2013). Because of this, clinical supervision is central to the process of lifelong learning and the benefits of clinical supervision outweigh its costs (Doncaster \& Bassetlaw Hospital, 2016). In the hospice setting, clinical supervision holds several challenges, with synchronising availability between clinical staff and facilitators being a significant factor within the inpatient unit.
Aims The aim of the project was to offer a meaningful clinical supervision resource to inpatient unit staff in line with the support being offered by the organisation to members of other teams. The Nursing \& Midwifery Council (NMC, 2018) states that clinical supervision should be available to registered nurses throughout their careers so they can constantly evaluate and improve their contribution to the care of people but identifying and testing a method which is workable for inpatient unit staff has been challenging, especially during a pandemic.

Methods

- Diarised group work with designated facilitators mixing members with varying job roles across the hospice with a generic scenario to discuss.

- Regular virtual sessions throughout the month organised by remote facilitator.

Results * Method 1 attendance (Q4). Two staff members over four hours clinical supervision.

* Method 2 attendance (Q4). 14 staff members over seven hours clinical supervision.

Conclusions It is clear to see from the above results that method 2 is a significantly more efficient way of engaging with staff than method 1 .

\section{EQUIPPING THE CARE HOME WORKFORCE FOR THE CHALLENGES ASSOCIATED WITH END-OF-LIFE CARE}

${ }^{1}$ Leonie Lowrie, ${ }^{2}$ Tracey Golding. ' Rennie Grove, Tring, UK; ${ }^{2}$ Peace Hospice Care, Watford, UK

\subsection{6/spcare-2021-Hospice.211}

Background We have a globally aging population that is going to impact on health and social care provision (Bone, Gomes, Etkind, et al., 2018); by 2040 care homes will be the most common place of death in the UK (Bone, Gomes, Etkind, et al., 2018). The burden of care is going to fall on the care home sector; it is vital that we equip them with the relevant knowledge and skill base to ensure equitable and excellent end-of-life care.

Aim To provide a sustainable approach in care homes for delivery of high standard of end-of-life care through education. To ensure excellent standards of holistic care at the endof-life.

Methods A structured 'Train the Trainer' educational package for care homes across two counties - provided by a collaboration of two hospices. This begins with a needs assessment and delivered through a blended learning approach:

- In person training.

- Discussion and case study.

- Personal reading and development.

- Virtual learning.

- Enquiry.

Followed by an audit to identify the learning and changes in practice. This will be supported by regular contact with educators.

Results Engagement with care homes across both counties, demonstrating effective training and ongoing support of champions. This will equip staff with a sustainable skill set and knowledge framework with a resulting change in end-of-life care which will meet the needs of our changing population (Bone, Gomes, Etkind, et al., 2018). This will include ongoing 